\title{
Novo surto de leishmaniose tegumentar americana em área de treinamento militar na Zona da Mata norte do Estado de Pernambuco
}

\author{
New outbreak of American tegumentary leishmaniasis in a military training center \\ in the Zona da Mata region, in the north of the State of Pernambuco
}

\author{
Maria Sandra Andrade, ${ }^{1,2}$, Maria Edileuza Felinto Brito', Salomão Thomaz da Silva ${ }^{2}$, \\ Edna Ishikawa ${ }^{3}$, Silvia Maria Santos Carvalho ${ }^{1}$ e Sinval Pinto Brandão-Filho ${ }^{1}$
}

\begin{abstract}
RESUMO
Relata-se novo surto de LTA em militares com 71 casos confirmados pelos critérios clínico, epidemiológico e laboratorial. Obteve-se o isolamento de sete amostras, identificadas como Leishmania (Viannia) braziliensis. A ocorrência de surtos nesta região confirma o caráter endêmico, cuja magnitude parece estar relacionada a não adoção de medidas de proteção individual.
\end{abstract}

Palavras-chaves: Leishmaniose tegumentar americana. Leishmania (Viannia) braziliensis. Ecopidemiologia. Mata Atlântica.

\begin{abstract}
A new outbreak of American tegumentary leishmaniasis among military personnel is reported, with 71 cases confirmed by means of clinical, epidemiological and laboratory criteria. Seven samples were isolated and were identified as Leishmania (Viannia) braziliensis. The occurrence of outbreaks in this region confirms the endemic nature of this disease, and the magnitude of the occurrence seems to be related to non-adoption of individual protection measures.
\end{abstract}

Key-words: American tegumentary leishmaniasis. Leishmania (Viannia) braziliensis. Eco-epidemiology. Atlantic rainforest.

No Estado de Pernambuco, o número de casos notificados de leishmaniose tegumentar americana (LTA) tem crescido nos últimos anos ${ }^{13}$. A região da Zona da Mata de Pernambuco responde por $64,2 \%$ do total de casos notificados 5 . É importante registrar que, a partir de 2000, verifica-se também um aumento da ocorrência de LTA no Sertão ${ }^{13}$, o que demonstra que a doença no estado apresenta não só um aumento no número de casos, como também uma importante expansão espacial.

Em 1996, foi documentado o primeiro surto de ITA no Campo de Instrução Marechal Newton Cavalcanti (CIMNC), com o registro de 26 casos autóctones em militares que realizaram treinamento na localidade. Um inquérito epidemiológico identificou uma prevalência de $24,1 \%$ de infecção nesta população $0^{4}$. A análise das notificações de 1996 a 2007 de casos de LTA em militares após treinamentos no CIMNC permite verificar um registro médio de 16 casos autóctones por ano. No entanto, observam-se picos no número de casos, seguidos de períodos de não ocorrência ou de ocorrência relativamente baixa de LTA na região.

\footnotetext{
1. Instituto Aggeu Magalhães, Fundação Oswaldo Cruz, Recife, PE. 2. Hospital Geral de Recife, Exército Brasileiro, Recife, PE, 3. Instituto Evandro Chagas, Belém, PA. Apoio Financeiro: CNPq, Projeto 410.481/2006-8.

Endereço para correspondência: Dr. Sinval Pinto Brandão-Filho. CPqAM/FIOCRUZ. Av. Moraes Rego s/n, Campus UFPE. 50670-420 Recife PE.

Tel: 5581 2101-2562

e-mail: sinval@cpqam.fiocruz.br

Recebido para publicação em 31/05/2009

Aceito em 15/09/2009
}

Surtos de LTA em militares, além da região de estudo ${ }^{34}$, têm sido relatados na região Norte do país ${ }^{9}$. A transmissão no CIMNC apresenta características epidemiológicas peculiares por se tratar de uma região com vasta área de mata primária remanescente e áreas fragmentadas de mata secundária. A relevância deste estudo está relacionada à necessidade de um maior entendimento das características da transmissão na localidade e também pela necessidade de desenvolvimento de estratégias e ferramentas mais efetivas de prevenção e controle da LTA ${ }^{10}$.

Este estudo, do tipo descritivo, relata a ocorrência de um surto de LTA, envolvendo militares que participaram de treinamento no CIMNC no período de julho a agosto de 2006. 0 Campo de Instrução Marechal Newton Cavalcanti localiza-se no município de Paudalho, Zona da Mata Norte do Estado de Pernambuco, a cerca de 40km do Recife, ocupando uma área de 6.280 hectares, com clima quente e úmido.

A população do estudo foi constituída por militares que participaram de treinamento no CIMNC em 2006. Os critérios de inclusão na amostra foram à participação em treinamentos nesta área e apresentar lesões suspeitas de LTA, após o seguimento de seis meses da realização do treinamento. Os militares que apresentaram lesões sugestivas de LTA realizaram teste de hipersensibilidade retardada (intradermorreação de Montenegro) e pesquisa direta do parasito. Foram realizadas biópsias nas lesões para o isolamento de Leishmania.

Amostras biológicas obtidas de biópsias e punções aspirativas de lesões cutâneas foram processadas no Laboratório de 
Imunoparasitologia do Instituto Aggeu Magalhães para isolamento do parasito, através da inoculação do material em meio de cultivo Agar Sangue Base ${ }^{7}$. As amostras também foram inoculadas em hamsters (Mesocricetus auratus), com o mesmo objetivo. A identificação ou tipagem dos flagelados foi realizada através de reações com um painel de vinte e três anticorpos monoclonais específicos (B2, B5, B12, B11, B13, B18, B19, C01, C02, C03, D13, L1, L12, M2, N2, N3, V1, WA2, W1, W2, WH1, WIC e T3) ${ }^{14}$.

Definiram-se como categorias de análise, os dados referentes à distribuição de participantes nos treinamentos militares, os resultados da IDRM, o diagnóstico e tratamento realizados. Os dados foram tabulados, utilizando-se o programa Excel/Microsoft Office e a análise descritiva foi realizada através da distribuição das frequências relativas.

0 trabalho foi aprovado na Comissão de Ética em Pesquisa do Instituto Aggeu Magalhães/FIOCRUZ.

Em 2006, 5.803 militares participaram de treinamentos militares no CIMNC, com duração média de 5 dias; 2.295 participaram de treinamentos em julho e agosto, destes 74 apresentaram lesões suspeitas de ITA (Tabela 1). Dos 74 casos considerados suspeitos para LTA, 71 (95,9\%) foram confirmados pelo critério clínico, epidemiológico e laboratorial e 100\% deles foram positivos ao teste de IDRM, com resultados variando de 6 a $23 \mathrm{~mm}$ de enduração. 0 exame parasitológico direto foi positivo em $47,9 \%(34 / 71)$ dos casos.

\section{TABELA 1}

Distribuição dos participantes de treinamentos e casos de leishmaniose tegumentar americana registrados no Campo de Instrução Marechal Newton Cavalcanti, Paudalho, Pernambuco, em 2006.

\begin{tabular}{lrrrrr}
\hline & \multicolumn{2}{c}{ Participantes } & & \multicolumn{2}{c}{ Casos } \\
\cline { 2 - 3 } \cline { 6 - 6 } Meses & $\mathrm{n}^{0}$ & FR $(\%)$ & & $\mathrm{n}^{0}$ & FR (\%) \\
\hline Abril & 1.220 & 21,0 & & - \\
Maio & 1.096 & 18,9 & & - \\
Junho & 193 & 3,3 & & - \\
Julho & 1.064 & 18,3 & & 10 & 14,1 \\
Agosto & 1.231 & 21,2 & & - & 85,9 \\
Setembro & 50 & 0,9 & & - \\
Outubro & 555 & 9,6 & & - \\
Novembro & 394 & 6,8 & & - \\
\hline Total & 5.803 & 100,0 & & 71 & 100,0 \\
\hline
\end{tabular}

FR: frequência relativa.

0 período médio de incubação foi de 33,6 dias; $92,9 \%$ (66/71) dos militares apresentavam lesão única e 7,1\% (5/71) duas lesões. Estas variavam de 0,3 a $2,5 \mathrm{~cm}$ de diâmetro e localizavam-se nas regiões da face, pavilhão auricular, mãos, região dos braços e antebraços, punho, pescoço. Dos militares acometidos por LTA, apenas 9,9\% (7/71) deles relataram o uso de repelente durante $o$ treinamento militar.

Os militares com diagnóstico confirmado foram tratados com antimoniato de N-metil glucamina (Glucantime $($ )), seguindo o esquema terapêutico de uma ampola/dia, aplicada por via intramuscular, durante 20 dias seguidos. Todos os militares apresentaram completa cicatrização das lesões após a conclusão do primeiro ciclo do tratamento.
Das 23 biópsias e punções aspirativas de lesões, obteve-se 7 isolados de formas compatíveis com Leishmania spp, que foram identificadas como Leishmania (Viannia) braziliensis, sorodemo 1, através do perfil de reações com anticorpos monoclonais específicos.

Os treinamentos militares são considerados atividade de risco para $\mathrm{LTA}^{8}$. 0 relato de casos da doença em militares após treinamento tem acontecido com frequência na Região Amazônica ${ }^{9}$. No CIMNC, após o primeiro surto investigado em $1996^{4}$, tem se verificado a ocorrência de um número importante de casos de LTA após os treinamentos militares ${ }^{1}$.

A forma cutânea localizada é a predominante e está associada à Leishmania (Viannia) braziliensis, o que se observa também nos achados verificados em outros estados da região Nordeste, como o Maranhão, Ceará, Bahia e também em outra área de Pernambuco ${ }^{3}$. Devemos ressaltar também que no seguimento de dez anos dos casos autóctones registrados no CIMNC, não foi verificada a forma mucosa da doença, como observada em Amaraji, na Zona da Mata Sul de Pernambuco ${ }^{3}$, e em Três Braços, Bahia ${ }^{11}$.

A resposta satisfatória ao tratamento com antimoniato de N-metil glucamina neste surto, e em outros casos na mesma regiã $0^{12}$, apresenta concordância com estudos que também obtiveram respostas terapêuticas satisfatórias, com apenas uma série de baixa dosagem desta droga de primeira escolha ${ }^{12}$. A busca ativa de casos de LTA, após treinamentos no CIMNC, possibilitando o diagnóstico precoce e tratamento imediato, provavelmente contribuiu para o sucesso do tratamento.

Considerando-se a série histórica de casos de LTA na região, verifica-se que se trata de uma importante área de transmissão ${ }^{1}$. No entanto, ressalta-se que, após a intensificação das atividades de prevenção e controle por parte das organizações militares (OM), em seguida ao surto de 1996, houve importante controle da LTA na região. Contudo, o relato dos militares acometidos de LTA sobre a não utilização de repelente durante os treinamentos e a presença de lesões em áreas dos antebraços e braços, regiões do corpo que poderiam estar protegidas pelo uniforme, permite-nos levantar a hipótese de ter havido descontinuidade das medidas de prevenção por parte das seções de saúde das $0 \mathrm{M}$ antes e durante a realização dos treinamentos no CIMNC, no período em que ocorreu este novo surto.

Ao contrário do que se observa em vários estudos no Brasil sobre a ecoepidemiologia da LTA, em relação à predominância do ciclo zoonótico, com os casos ocorrendo em áreas desmatadas e de colonização antiga, e com vetores de hábitos domiciliares e peridomiciliares, a ocorrência deste novo surto no CIMNC, associada aos achados prévios ${ }^{14}$, reforçam as evidências da manutenção do ciclo enzoótico, com o surgimento de casos após treinamentos. A magnitude dos novos casos parece estar relacionada com a não observação das medidas de proteção individual pelos militares durante a realização dos treinamentos.

A análise do panorama atual sobre surtos e a expansão da LTA revelam a necessidade de novas reflexões sobre as implicações 
da heterogeneidade do quadro ecoepidemiológico evolutivo da LTA em relação à necessidade de se obter recursos profiláticos mais eficientes para controlar a transmissão da doença ${ }^{10}$. 0 aprimoramento dessas ações depende também de mais estudos voltados para o melhor conhecimento da biologia de reservatórios e vetores envolvidos na cadeia de transmissão, possibilitando também esclarecer a história natural da LTA associada à Leishmania (Viannia) braziliensis.

Como forma de controle para situações como a do CIMNC, sugere-se que os treinamentos militares sejam planejados de forma a ocorrerem em períodos nos quais estudos na região ${ }^{24}$ evidenciaram uma menor incidência de vetores e de casos humanos, o que possibilitaria a minimização do contato homemvetor com possível redução do número de casos.

Ressalta-se ainda a necessidade da utilização de medidas de proteção individual e a realização de palestras educativas sobre LTA, como uma rotina permanente de procedimentos a serem adotados por ocasião dos treinamentos e retorno dos militares às suas organizações militares, para que não ocorra descontinuidade das atividades de prevenção e controle.

\section{AGRADECIMENTOS}

Ao CNPq pelo suporte financeiro, e ao comando da Sétima Região Militar do Exército Brasileiro, pelo apoio logístico.

\section{REFERÊNCIAS}

1. Andrade MS, Brito MEF, Silva ST, Lima BS, Almeida EL, Albuquerque EL, Marinho Jr JF, Ishikawa E, Cupolillo E, Brandão-Filho SP. Leishmaniose tegumentar americana causada por Leishmania (Viannia) braziliensis, em área de treinamento militar na Zona da Mata de Pernambuco. Revista da Sociedade Brasileira de Medicina Tropical 38:229-233, 2005.

2. Andrade MS, Valença HF, Silva AL, Almeida FA, Almeida EL, Brito MEF, Brandão-Filho SP. Sandfly fauna in a military training area for American tegumentary leishmanisis in the Atlantic rain forest region of Pernambuco State, Brazil. Cadernos de Saúde Pública 21:1761-1767, 2005.
3. Brandão-Filho SP. Ecoepidemiologia da leishmaniose tegumentar americana associada à Leishmania (Viannia) braziliensis na Zona da Mata Atlântica do Estado de Pernambuco, Brasil. Tese de Doutorado. Universidade de São Paulo. São Paulo, 2001.

4. Brandão-Filho SP, Brito MEF, Martins CAP, Sommer IB, Valença HF, Almeida FA, Gomes J. Leishmaniose tegumentar americana em centro de treinamento militar localizado na Zona da Mata de Pernambuco, Brasil. Revista da Sociedade Brasileira de Medicina Tropical 31:575-576, 1998.

5. Brandão-Filho SP, Campbell-Lendrum DH, Brito MEF, Shaw JJ, Davies CR. Epidemiological surveys confirm an increasing burden of cutaneous leishmaniasis in north-east Brazil. Transactions of the Royal Society of Tropical Medicine and Hygiene 93:488-494, 1999.

6. Costa JML, Balby ITA, Rocha EJSR, Silva AR, Rebêlo JMM, Ferreira LA. Estudo comparativo da leishmaniose tegumentar americana em crianças e adolescentes procedentes das áreas endêmicas de Buriticupu (Maranhão) e Corte de Pedra (Bahia), Brasil. Revista da Sociedade Brasileira de Medicina Tropical 31:279-288, 1998.

7. Evans D. Handbook on isolation characterization and cryopreservation of leishmania. World Heath Organization, Geneva, Switzerland, 1989.

8. Falqueto A, Sessa PA. Leishmaniose Tegumentar Americana. In: Focaccia R (org) Tratado de infectologia. Editora Atheneu, São Paulo, p.1543-1557, 2005.

9. Guerra JAO, Talhari S, Paes MG, Garrido M, Talhari JM. Aspectos clínicos e diagnósticos da leishmaniose tegumentar americana em militares simultaneamente expostos à infecção na Amazônia. Revista da Sociedade Brasileira de Medicina Tropical 36:587-590, 2003.

10. Marzochi MCA. Leishmanioses no Brasil: As leishmanioses tegumentares. Jornal Brasileiro de Medicina 63: 82-104, 1992.

11. Marsden PD. Personal experience with diagnostic and therapeutic aspects of human Leishmania (Viannia) Braziliensis in Tres Braços. Memórias do Instituto Oswaldo Cruz 89: 485-487, 1994.

12. Oliveira-Neto MP, Schubah A, Mattos M, Gonçalves-Costa SC, Pirmez C. A low dose antimony treatment in 159 patients with american cutaneous leishmaniasis: extensive follow-up studies (up to 10 years). The American Journal of Tropical Medicine Hygiene 57:651-655, 1997.

13. Secretaria de Saúde do Estado de Pernambuco. Leishmaniose tegumentar americana em Pernambuco. Diretoria de Informação da Secretaria Estadual de Saúde. Boletim Epidemiológico. a.1 nº 3. 2002.

14. Shaw JJ, Ishikawa E, Lainson R. A rapid and sensitive method for the identification of Leishmania with monoclonal antibodies using fluorescein-labelled avidin. Transactions of the Royal Society of Tropical Medicine and Hygiene 83:783-784, 1989 